\title{
TITLE:
}

\section{Heterogeneity in Polymer Thin Films}

$\operatorname{AUTHOR}(\mathrm{S})$ :

Kanaya, Toshiji; Inoue, Rintaro; Nishida, Koji

\section{CITATION:}

Kanaya, Toshiji ...[et al]. Heterogeneity in Polymer Thin Films. AIP Conference Proceedings 2011, 1349: 23-26

\section{ISSUE DATE:}

2011

URL:

http://hdl.handle.net/2433/160668

\section{RIGHT:}

Copyright 2011 American Institute of Physics. This article may be downloaded for personal use only. Any other use requires prior permission of the author and the American Institute of Physics. The following article appeared in AIP Conference Proceedings 1349, 23 (2011) and may be found at http://link.aip.org/link/?apc/1349/23 


\title{
Heterogeneity in Polymer Thin Films
}

\author{
Toshiji Kanaya, Rintaro Inoue and Koji Nishida
}

\author{
Institute for Chemical Research, Kyoto University, Uji, Kyoto-fu 611-0011, Japan
}

\begin{abstract}
In the last two decades very extensive studies have been performed on polymer thin films to reveal very interesting but unusual properties. One of the most interesting findings is the decrease in glass transition temperature Tg with film thickness in polystyrene (PS) thin film supported on Si substrate. Another interesting finding is apparent negative thermal expansivity in glassy state for thin films below $\sim 25 \mathrm{~nm}$. In order to understand the unusual properties of polymer thin films we have studied temperature dependence of thickness of polystyrene thin films by means of X-ray and neutron reflectivity. In addition, we also studied dynamics of PS thin films using inelastic neutron scattering. In the presentation we will discuss the results from viewpoints of heterogeneity of polymer thin films. Finally we did neutron reflectivity measurements on a 5-layer thin film, consisting of alternatively stacked d-PS and h-PS layers to see the distribution of glass transition temperature and thermal expansivity directly.
\end{abstract}

Keywords: Polymer thin film, glass transition, X-ray reflectivity, Neutron reflectivity, Inelastic neutron reflectivity, heterogentiy.

PACS: 64.70.pj, 68.60.Dv, 61.05.fj

\section{INTRODUCTION}

Recent extensive studies on polymer thin films have revealed very interesting but unusual properties of polymer thin films. Glass transition of thin films is one of the most interesting topics in the field because many properties such as mechanical and thermal properties change drastically at the glass transition temperature $T_{\mathrm{g}}$. In a pioneering work by Keddie et al. [1] it was reported that the glass transition temperature of polystyrene thin films supported on silicon substrate decreases with decreasing thickness below $\sim 40 \mathrm{~nm}$. Similar results were reported by many researchers on $T_{\mathrm{g}}$ of polystyrene thin films supported on silicon substrate. On the other hand, $T_{\mathrm{g}}$ increases with decreasing the film thickness for poly(methyl methacrylate)(PMMA) supported on silicon substrate [2], suggesting strong interactions between them. Thus, surface and/or interfacial effects seem to play an important role on glass transition. In order to reveal the cause of the decrease in $T_{\mathrm{g}}$ Kajiyama et al. [3-5] have studied $T_{\mathrm{g}}$ of the surface layer of polystyrene thin films to find a lower glass transition temperature $T_{\mathrm{g}}$, and concluded that as the film thickness decreases the fraction of the surface layer increases, resulting in the reduction in $T_{\mathrm{g}}$. This is very normal explanation for the reduction in $T_{\mathrm{g}}$ for thin films. Another interesting finding is the reduction of thermal expansivity with film thickness, which was first reported by NIST group [6]. It was revealed that one of the causes of the reduction was structure relaxation during the heating due to lack of annealing. However, the annealing experiments on thin films thickness [7] showed that the thermal expansivity decreased with film thickness even after long annealing above $T_{\mathrm{g}}$. This could be interpreted in terms of hard layer near the substrate, suggesting heterogeneous structure of polymer thin films.

It should be noted that polymers have wide distribution of the relaxation time in time scale as well as in length scale. Therefore, in order to understand the unusual properties of tin films we have to reveal the distribution of $T_{\mathrm{g}}$ as well as the dynamic nature. In other words, revealing heterogeneous structure and/or dynamics in polymer thin films is essential to understand the unusual properties. This has been already pointed out by de Gennes [8], and he mentioned that further experiments should not aim at the determination of a single $T_{\mathrm{g}}$, but at a distribution of $T_{\mathrm{g}}$ 's. Recent woks by Torkelson et al. [9] along the direction are remarkable. Using a fluorescence technique they examined glass transition temperature at surface, interface and in between of multilayer films.

In this report we studied the dynamics of polymer thin films by inelastic neutron scattering, and the distribution of glass transition temperature $T_{\mathrm{g}}$ in a multi-layer polystyrene thin film by neutron 
reflectivity, aiming to elucidate the heterogeneous structure or multi-layer structure of polymer thin films.

\section{EXPERIMENTAL}

The sample used for the inelastic scattering experiments was PS with molecular weight $M_{w}=$ $2.90 \times 10^{5}$ and molecular weight distribution $M_{w} / M_{n}=1.06$. Thin films were prepared by spin-coating toluene solutions of PS at $2000 \mathrm{rpm}$ on flat glass plates rinsed in toluene prior to spin-coating. The film was removed from the glass plate onto water surface and then collected on $\mathrm{Al}$ foil $15 \mathrm{~mm}$ thick, and then annealed at $413 \mathrm{~K}$ for $12 \mathrm{~h}$ after drying in vacuum at room temperature for 2 days. We used 300 sheets for both 1000 and $200 \AA$ films. For neutron reflectivity (NR) measurements we used deuterated polystyrene (d-PS) and hydrogenated polystyrene (h-PS) with molecular weight $M_{\mathrm{w}}=7.31 \times 10^{5}$ and $M_{\mathrm{w}}=7.69 \times$ $10^{5}$, and molecular weight distributions were $M_{\mathrm{w}} / M_{\mathrm{n}}=$ 1.18 and 1.08 , respectively. The sample was annealed at $408 \mathrm{~K}$ for $5 \mathrm{~min}$ after drying in vacuum at $343 \mathrm{~K}$ for 2 days.

Inelastic neutron scattering measurements were performed with two spectrometers. One is an inverted geometry time of flight (TOF) spectrometer LAM-40 with an energy resolution of $\sim 0.2 \mathrm{meV}$, KEK, Tsukuba. Another is a direct geometry spectrometer MARI with an energy resolution $0.34 \mathrm{meV}$ at ISIS, Rutherford Appleton Laboratory, Didcot.

Neutron reflectivity (NR) measurements were performed using MINE spectrometer at JRR-3 reactor in Tokai, and ARISA-II at BL16 in J-PARC in Tokai.

\section{RESULTS AND DISCUSSION}

Inelastic neutron scattering measurements on the PS thin films were done in a temperature range below and above the glass transition temperature $T_{\mathrm{g}}$. First we have evaluated the mean square displacement $\left\langle u^{2}\right\rangle$ from the $Q$ dependence of the elastic scattering intensity. The evaluated $\left\langle u^{2}\right\rangle$ at $230 \mathrm{~K}$ is shown in Fig. 1 as a function of film thickness. It is clear that the $\left\langle u^{2}\right\rangle$ decreases with film thickness. Analyzing the inelastic scattering part of the spectra for the thin films we also found that the density of phonon states $G(\omega)$ decreases with the film thickness according to the reduction in $\left\langle u^{2}\right\rangle$. Both of the findings suggest that some hardening occurred in the thin film. We considered two possibilities for the decrease in $\left\langle u^{2}>\right.$ or the hardening with film thickness. One is the hardening of polymer chains due to the spatial confinement (confinement effect). Another possibility is that a hard layer (lower mobility layer) would exist at the interface between the polymer thin film and the

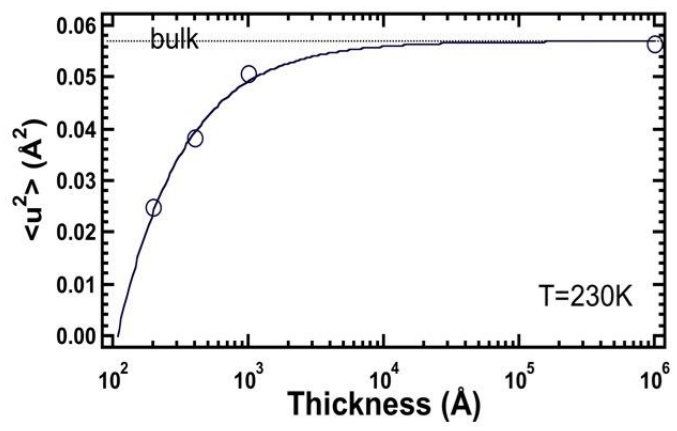

Fig. 1 Mean square displacement $<\mathrm{u} 2>$ of PS thin films at $230 \mathrm{~K}$ as a function of film thickness.

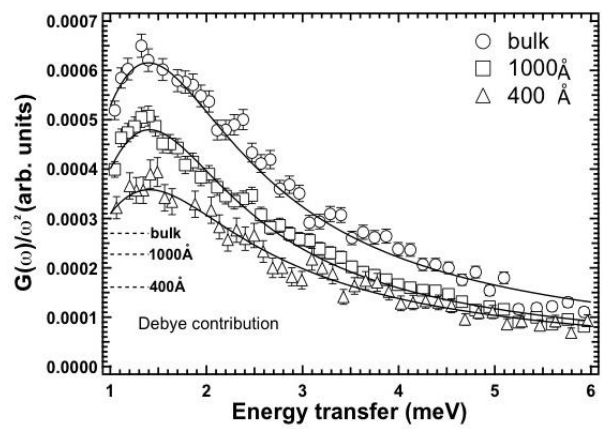

Fig. 2. Density of phonon states G( $\omega)$ for PS bulk, thin films $1000 \AA$ and $400 \AA$ thick. Dashed lines show the Debye contribution.

substrate. In order to see which cause is more plausible, we prepared thin films $40 \mathrm{~nm}$ thick for different molecular weights $\left(M_{w}\right)$, giving different ratios of the film thickness $(=d)$ to the double radius of gyration $\left(2 R_{g}\right)$. If the confinement effect were dominant, the decrease of $\left\langle u^{2}\right\rangle$ would be observed with the decrease of ratio of $d / 2 R_{g}$ because decreasing the ratio $d / 2 R_{g}$ means an increase of spatial confinement. And if the interface effect were dominant, $\left\langle u^{2}\right\rangle$ would be constant regardless of the ratio $d / 2 R_{g}$ because the ratio is constant under the same film thickness. We found [10] that $\left\langle u^{2}\right\rangle$ was independent of molecular weight, at least, within the experimental error. We therefore concluded that the decrease in $\left\langle u^{2}\right\rangle$ is caused due to the hard layer near the substrate, suggesting heterogeneous structure in the thin films.

We also studied the non-Gaussian parameter $A_{0}$ of polymer thin films to study the dynamic heterogeneity [11]. We found that the non-Gaussian parameter $A_{0}$ increased with decreasing the film thickness (Fig. 3), showing the dynamic heterogeneity increased with decreasing the film thickness, again suggesting that the polymer thin films have heterogeneous structure. 


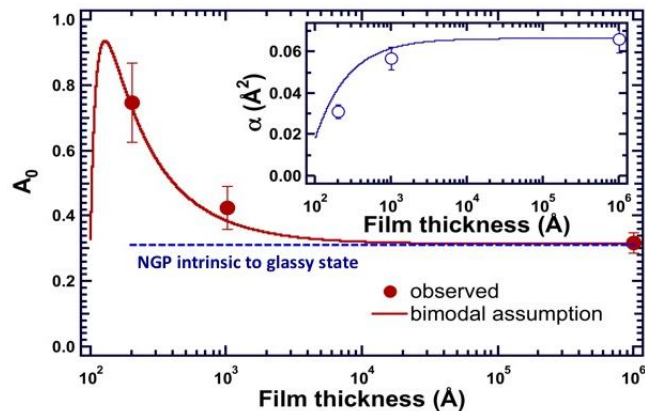

Fig. 3. Non-Gaussian parameter as a function of film thickness at $230 \mathrm{~K}$. Inset shows the mean square displacement for PS thin films.

Next we performed neutron reflectivity measurements on a 5-layer thin film, consisting of alternatively stacked d-PS and h-PS layers (see Fig. 4). Each layer is $\sim 20 \mathrm{~nm}$ thick and the total thickness is $\sim 100 \mathrm{~nm}$. We have analyzed the reflectivity profiles to evaluate thickness of each layer and interface and surface roughness as a function of temperature. The temperature dependence of the total film thickness is shown at top in Fig. 5. The total film thickness increases with temperature in the glassy state up to $\sim 372 \mathrm{~K}$ and begins to increase more steeply above $\sim 372 \mathrm{~K}$, showing the glass transition temperature $T_{\mathrm{g}}$ is $\sim 372 \mathrm{~K}$, which is almost the same as the bulk glass transition temperature. It is noted that in

\begin{tabular}{|c|}
\hline deutrated polystyrene (d-PS) \\
\hline hydrogenated polystyrene (h-PS) \\
\hline deutrated polystyrene (d-PS) \\
\hline hydrogenated polystyrene (h-PS) \\
\hline deutrated polystyrene (d-PS) \\
\hline substrate \\
\hline
\end{tabular}

Fig. 4 Schematic representation of 5-layer thin film consisting of alternatively stacked d-PS and h-PS layers.

this thin film we did not observe the decrease in thickness slightly below $T_{\mathrm{g}}$, which was typically observed in not-well annealed thin films [7] and assigned to structure relaxation due to lack of annealing. The present observation suggests that the annealing condition in this experiment is enough to avoid the structure relaxation near $T_{\mathrm{g}}$. The temperature dependences of the thickness for the $1^{\text {st }}, 2^{\text {nd }}, 3^{\text {rd }}, 4^{\text {th }}$ and more steeply, showing that the glass transition temperature of the surface layer is $\sim 20 \mathrm{~K}$ lower than $5^{\text {th }}$ layer from top (surface)to bottom are shown in Fig. 5. The surface layer increases in thickness with temperature, while it begins to increase above $\sim 356 \mathrm{~K}$

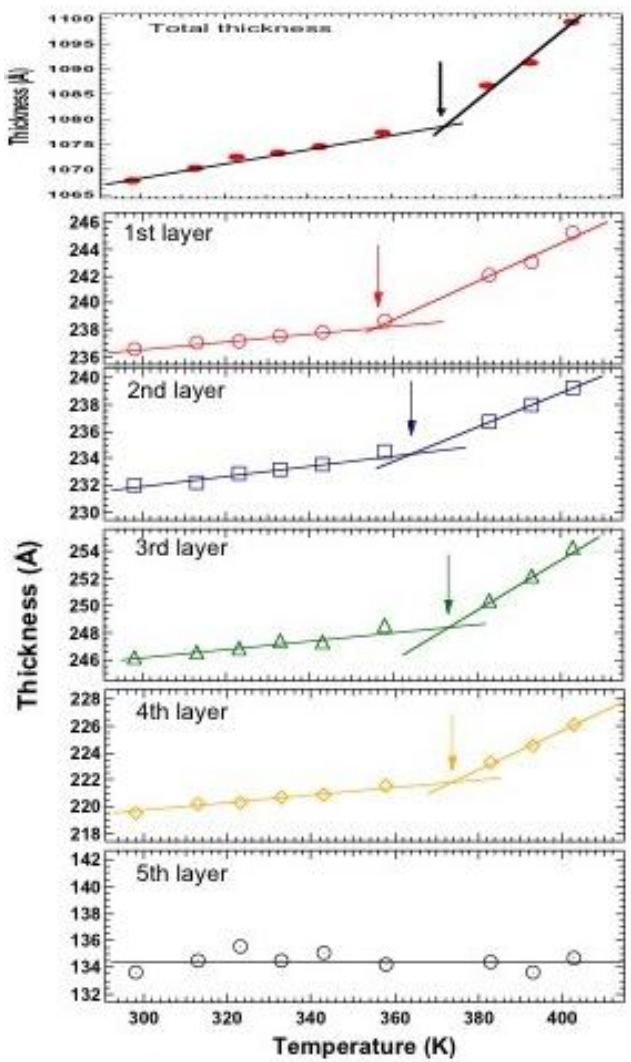

Fig. 5 Temperature dependence of film thickness of each layer.

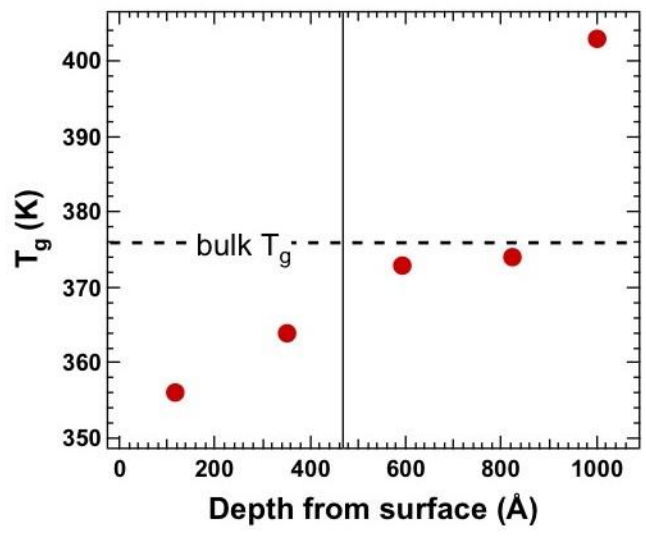

Fig. 6 Distribution of glass transition temperature $\mathrm{Tg}$ in 5-layer thin film

the bulk $T_{\mathrm{g}}$. The glass transition temperature $T_{\mathrm{g}}$ increases with departing from the surface. The $T_{\mathrm{g}}$ 's of the $3^{\text {rd }}$ and $4^{\text {th }}$ layers are almost the same as that of bulk. On the other hand, the bottom layer ( $5^{\text {th }}$ layer) 
shows very different temperature dependence of the thickness from other layers. The film thickness is almost independent of temperature below $403 \mathrm{~K}$. In the temperature range examined we did not observe the increase in thickness, suggesting that the glass transition temperature of the bottom layer must be higher than $403 \mathrm{~K}$. The present results clearly show that the thin film has a distribution of $T_{\mathrm{g}}$ (Fig. 6) even though the $T_{\mathrm{g}}$ of the total thickness is the same as the bulk $T_{\mathrm{g}}$.

\section{REFERENCES}

1. J. L. Keddie, R. A. Jones and R. A. Cory, Europhys. Lett., 27, 59 (1994)

2. J. L. Keddie, R. A. Jones and R. A. Cory, Faraday Discuss., 98, 219 (1994)

3. T. Kajiyama, K. Tanaka, N. Satomi and A. Takahara, Macromolecules, 31, 5150 (1998)
4. T. Kajiyama, K. Tanaka and A. Takahara, Polymer, 39, 4665 (1998)

5. T. Kajiyama, K. Tanaka and A. Takahara, Macromolecules, 32, 4474 (1999)

6. W. J. Orts, J. H. vanZanten, W. W.-1. Wu and S. K. Satija, Phys. Rev. Lett., 71, 867 (1993).

7. T. Miyazaki, K. Nishida and T. Kanaya, Phys. Rev. E, 69, 022801 (2004)

8. R. Inoue, T. Kanaya, K. Nishida, I. Tsukushi, and K. Shibata, Phys. Rev. Lett., 95, 056102 (2005). R. Inoue, T. Kanaya, K. Nishida, I. Tsukushi, and K. Shibata, Phys. Rev. E, 74, 021801 (2006).

9. P. G. de Gennes Eur. Phys. J. E, 2, 201 (2000)

10. R. D. Priestley, C. J. Ellison, L. J. Broadbelt and J. M. Torkelson, Science, 309, 456 (2005)

11. R. Inoue, T. Kanaya, K. Nishida, I. Tsukushi, and K. Shibata, Phys. Rev. E, 74, 021801 (2006). 
Copyright of AIP Conference Proceedings is the property of American Institute of Physics and its content may not be copied or emailed to multiple sites or posted to a listserv without the copyright holder's express written permission. However, users may print, download, or email articles for individual use. 